shape muscle gene expression and regulate sarcomeric actin organization. Genes Dev. 23:619-632.

25. Rao, P.K., Kumar, R.M., Farkhondeh, M., Baskerville, S., and Lodish, H.F. 2006. Myogenic factors that regulate expression of muscle-specific microRNAs. Proc. Natl. Acad. Sci. U. S. A. 103:8721-8726.

26. Sharp, R., et al. 2002. Synergism between INK4a/ $\mathrm{ARF}$ inactivation and aberrant $\mathrm{HGF} / \mathrm{SF}$ signaling in rhabdomyosarcomagenesis. Nat. Med. 8:1276-1280.

27. Anastasi, S., et al. 1997. A natural hepatocyte growth factor/scatter factor autocrine loop in myoblast cells and the effect of the constitutive Met kinase activation on myogenic differentiation. J. Cell Biol. 137:1057-1068.
28. Adams, B.D., Cowee, D.M., and White, B.A. 2009. The role of miR-206 in the epidermal growth factor (EGF) induced repression of estrogen receptoralpha (ER \{alpha\}) signaling and a luminal phenotype in MCF-7 breast cancer cells. Mol. Endocrinol. Online publication ahead of print. doi:10.1210/ me.2009-0062.

29. Wurdinger, T., and Costa, F.F. 2007. Molecular therapy in the microRNA era. Pharmacogenomics J. 7:297-304.

30. Bumcrot, D., Manoharan, M., Koteliansky, V., and Sah, D.W. 2006. RNAi therapeutics: a potential new class of pharmaceutical drugs. Nat. Chem. Biol. 2:711-719.
31. Wang, V., and Wu, W. 2009. MicroRNA-based therapeutics for cancer. BioDrugs. 23:15-23.

32. Mishra, P.J. 2009. MicroRNA polymorphisms: a giant leap towards personalized medicine. Personalized Medicine. 6:119-125. doi:10.2217/17410541.6.2.119.

33. Ohno, R., et al. 1993. Multi-institutional study of all-trans-retinoic acid as a differentiation therapy of refractory acute promyelocytic leukemia. Leukaemia Study Group of the Ministry of Health and Welfare. Lenkemia. 7:1722-1727.

34. Labourier, E., et al. 2009. microRNAs as potential diagnostic and prognostic markers of disease. http://www.ambion.com/techlib/posters/ miRNA_0405.html.

\title{
Genomic amplicons target vesicle recycling in breast cancer
}

\author{
Gordon B. Mills, ${ }^{1}$ Igor Jurisica, ${ }^{2}$ Yosef Yarden, ${ }^{3}$ and Jim C. Norman ${ }^{4}$
}

\author{
${ }^{1}$ Department of Systems Biology, University of Texas MD Anderson Cancer Center, Houston, Texas, USA. \\ ${ }^{2}$ Division of Signaling Biology, Ontario Cancer Institute, Toronto, Ontario, Canada. ${ }^{3}$ Department of Biological Regulation, \\ Weizmann Institute of Science, Rehovot, Israel. ${ }^{4}$ Integrin Cell Biology Laboratory, Beatson Institute for Cancer Research, Glasgow, United Kingdom.
}

\begin{abstract}
Aberrant endocytosis, vesicle targeting, and receptor recycling represent emerging hallmarks of cancer. In this issue of the JCI, Zhang and colleagues demonstrate that RAB-coupling protein $(R C P$; also known as $R A B 11 F I P 1)$ is a "driver" of the 8p11-12 amplicon in human breast cancer and mouse xenograft models of mammary carcinogenesis (see the related article beginning on page 2171). Their finding that RAB GTPase function enables genomic amplification to confer aggressiveness to mammary tumors adds significantly to the body of evidence supporting pivotal roles for receptor trafficking in the proliferation and metastasis of cancer.
\end{abstract}

\section{$R C P$ is a potential driver of the 8p11-12 amplicon in breast cancer}

DNA copy number aberrations often increase the number of copies of key genes that can drive cancer progression. However, despite improved approaches for mapping regions of copy number increases (known as amplicons), the complex structure and large size of most amplification events make it challenging to identify true "driver" genes.

In this issue of the JCI, Zhang and colleagues apply a novel algorithm, termed TRIAGE (triangulating oncogenes through clinico-genomic intersects), to a collection of microarray expression profiles of

Conflict of interest: G.B. Mills receives research support from Celgene, Exelixis, GlaxoSmithKline, and Lpath Inc. The authors have declared that no other conflict of interest exists.

Nonstandard abbreviations used: RCP, RAB-coupling protein; RTK, receptor tyrosine kinase; TRIAGE, triangulating oncogenes through clinico-genomic intersects.

Citation for this article: J. Clin. Invest. 119:2123-2127 (2009). doi:10.1172/JCI40256. primary human breast cancers in an effort to identify candidate genes in amplicons that could contribute to patient outcome (1). The TRIAGE algorithm is based on the concept that transcript levels of genes located in amplicons are frequently coordinately elevated. Thus, by mapping RNA levels onto the chromosome, genomic regions deranged by amplicons can be identified. The authors' application of TRIAGE identified a $1-\mathrm{Mb}$ region contiguous with the well-characterized 17q12 amplicon, which is known to harbor multiple genes, including the receptor tyrosine kinase (RTK) ERBB2 (also known as HER2 and NEU), that are associated with breast cancer development and progression (2). This region was the strongest "hit" returned, supporting the contention that the TRIAGE algorithm provides a novel approach to identifying candidate genes within amplicons that contribute to tumorigenesis.

Using this same approach, Zhang et al. (1) also identified the gene RAB11 family-interacting protein 1 (RAB11FIP1; also known and herein referred to as RAB-cou- pling protein $[R C P])$ as a candidate driver of the 8p11-12 amplicon, which is present in $10 \%-25 \%$ of breast cancers and is strongly associated with breast cancer subtype and outcome $(3,4) . R C P$ is an effector and binding partner of the RAB11 family (including RAB11A, RAB11B, and RAB25) of RAB small GTPases that control vesicle recycling. The studies by Zhang et al. (1) demonstrating that $R C P$ (8p11-12) is overexpressed as a consequence of genomic amplification, combined with previous studies of genomic amplicons including RAB25 (1q22) (5) and RAB23 (6p11; ref. 6), suggest that genomic amplicons frequently target vesicle function in cancer.

\section{$R C P$ exhibits the characteristics of an oncogene}

Zhang et al. performed detailed functional studies to determine whether $R C P$ has oncogene-like characteristics (1). Based on transfection and knockdown studies, they found that $R C P$ is not sufficient to transform naive cells. However, in breast cancer cell lines, $R C P$ decreased growth factor dependence; increased survival under anoikis conditions and induced motility, invasion, and epithelial-mesenchymal transition (EMT) in vitro; and increased tumor growth and progression in vivo, compatible with RCP being a key regulator of tumor aggressiveness.

The authors further show that RCP could be coprecipitated with the H-RAS proto- 
Table 1

RAB11FIPs are overexpressed and implicated in the pathophysiology of a number of cancer lineages

\begin{tabular}{|c|c|c|c|c|c|c|c|c|c|}
\hline Tumor type & $R C P$ & RAB11FIP2 & RAB11FIP3 & RAB11FIP4 & RAB11FIP5 & $R A B 11 A$ & $R A B 11 B$ & RAB25 & RAB23 \\
\hline Breast & $10^{-8}$ & & & & $10^{-6}$ & $10^{-8}$ & & $10^{-38}$ & \\
\hline Colon & $10^{-50}$ & & & $10^{-16}$ & & $10^{-17}$ & & $10^{-50}$ & \\
\hline Lung & $10^{-11}$ & & & & & & & $10^{-24}$ & \\
\hline Ovarian & & $10^{-11}$ & & $10^{-17}$ & & & & & \\
\hline Renal & & $10^{-12}$ & $10^{-42}$ & & $10^{-50}$ & & & & \\
\hline Endometrial & & $10^{-18}$ & & $10^{-20}$ & & & & $10^{-17}$ & $10^{-13}$ \\
\hline Prostate & & & & & & $10^{-11}$ & & $10^{-10}$ & $10^{-22}$ \\
\hline Bladder & & & & & & & & $10^{-10}$ & \\
\hline Carcinoid & & & $10^{-13}$ & & & & & & \\
\hline
\end{tabular}

Aberrations with $P \leq 10^{-6}$ are indicated. Data are derived from a reanalysis of Bittner_Multi-cancer through Oncomine Research (www.oncomine.org).

oncogene and that RCP increased H-RAS activity and markedly increased activation of the downstream target MAPK, suggesting a potential mechanism of action for the oncogenic effect of RCP (1). Strikingly, these effects of RCP were specific for H-RAS, with limited effects on the K-RAS or N-RAS protooncogenes. RAB11A and RAB25 are both partners for RCP. RCP promotes recycling of EGFR1 in a way that influences its signaling to PKB/AKT and MAPKs within endosomes (7). Since RCP, RAB11A, RAB25, H-RAS, EGFR, and components of their downstream signaling pathways colocalize in endosomes, the ability to coprecipitate RCP and H-RAS may reflect residency in a common endosomal compartment rather than a direct functional association.

\section{RCP and its binding partners are aberrant in cancer}

Germline mutations in RAB family members have been implicated in a number of hereditary diseases (see ref. 8 for review). However, mutations in RABs and their binding proteins have not been identified in a significant proportion of cancers. Intriguingly, the p85 subunit of the PI3K complex that acts as a RAB GTPase-activating protein, albeit with weak activity toward RAB11 (9), is mutated in a significant number of gliomas and rarely in other cancer lineages. Although the underlying mechanisms are unknown in most cases, many RAB family members and RAB11FIPs are overexpressed and thus implicated in the pathophysiology of particular cancer lineages (8) (Table 1). Indeed, mRNA levels of RCP and RAB25 are highly correlated in breast cancer samples (reanalysis of data in ref. 4), indicating that these two genes may cooperate with one another during tumorigenesis. $R A B 11 B$, in contrast with the other RAB11 family members, is not selectively overexpressed in any cancer lineage (Table 1), adding credence to the concept that functions mediated by the other family members are selected during tumor initiation or progression.

Zhang et al. demonstrated that RCP was the only RAB11FIP family member whose RNA correlated with disease progression in breast cancer (1). RAB25 is increased in hormone receptor-positive and HER2-positive tumors (5) and may indeed be decreased in basal breast cancers and selectively inhibit the function of this tumor lineage (10). Further, RAB11A expression is elevated in ductal carcinoma in situ (DCIS) and contributes to altered cellular outputs (11). Thus, the functions of RCP and its binding partners are likely required in different contexts during breast cancer development.

\section{Functions of RAB proteins}

When activated receptors are internalized from the cell surface, they are delivered to early endosomes where key decisions are made as to whether receptors are sent to late endosomes for degradation, directed to other subcellular compartments, or are returned (or recycled) to the plasma membrane to provide additional receptors for activation. Recycling receptors may be transported from early endosomes to the endosomal recycling compartment (which is commonly located in the perinuclear region) and returned to the plasma membrane in a RAB11/RAB25-dependent manner (Figure 1). Transit through the endosomal pathway is required to generate important cellular signals. For example, activity of cell-surface receptors such as EGFR and PDGFR is regulated within the endocytic compartment by selective association with ligands or functional regulators that does not occur at the cell membrane $(12,13)$. Indeed, association of cell-surface receptors such as EGFR and integrins can occur in intracellular vesicles, and this has been shown to influence the propagation of their downstream signals (7) (Figure 1). By demonstrating that RCP is required for growth and metastasis of breast xenografts in mice, the studies of Zhang et al. (1) have identified a critical genetic change occurring in breast cancer that most likely drives cancer progression by reconfiguring the way in which receptors, including integrins and RTKs, are trafficked intracellularly and recycled to the cell surface.

\section{RCP and its binding proteins are selectively involved in motility and invasion}

RAB-dependent trafficking of adhesion receptors (integrins) and RTKs influences cell migration and the generation and maintenance of cell polarity as cells undergo movement. Receptor recycling is now thought to contribute to cell migration/polarity, not by moving membrane en masse from the back to the front of cells, but by restricting receptor distribution by continuously retargeting the receptors back to the regions from which they were internalized. This, in turn, spatially restricts and sustains downstream signaling (Figure 1B). Given that the consequence of such localized signaling is, in many cases, the activation of Rho subfamily GTPases, it is not difficult to envisage how this then influences local cytoskeletal dynamics to drive cancer cell invasion or to maintain polarity.

RCP influences $\beta_{1}$ integrin-dependent cancer cell migration in both $2 \mathrm{D}$ and $3 \mathrm{D}$ contexts (7). Furthermore, when cells migrate in $3 \mathrm{D}$ matrices, the ability of RCP 


\section{A Tumor cell}

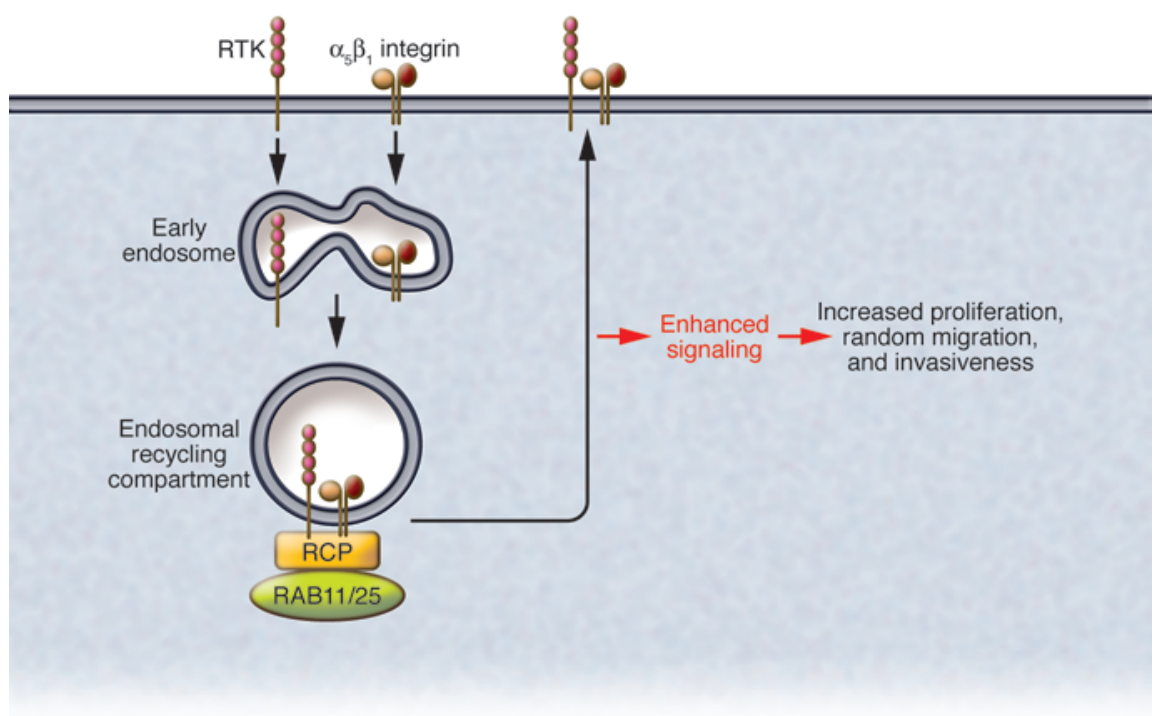

B Invasive tumor cell

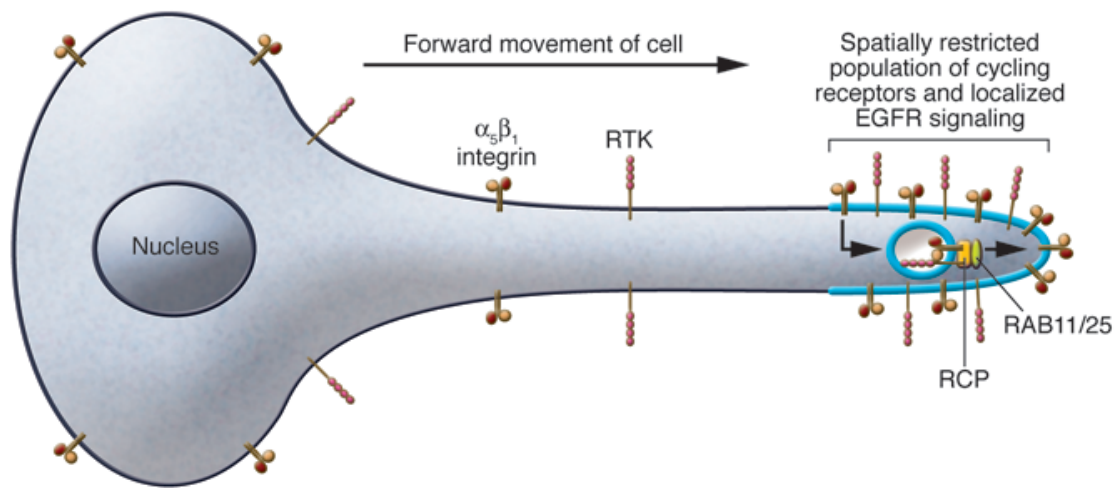

and its binding partner RAB25 to localize integrin and EGFR signaling to the cell front drives the extension of invasive pseudopods (ref. 14; Figure 1B). This trafficking-dependent localization of signaling proteins such as $\beta_{1}$ integrin and EGFR may contribute to the role of RCP in metastasis demonstrated by Zhang et al. (1). Notwithstanding, there are differences among the results of studies in regard to the profile of downstream signaling that is promoted by RCP. In contrast to the Zhang et al. study (1), in which RCP was shown to increase RAS/ERK signaling, our studies have demonstrated that both RCP and RAB25 selectively enhance PKB/AKT activity (5, 7). It is interesting to speculate as to the reasons that might underlie this discrepancy. The breast cancer cell lines (MCF7, MCF10A, and MDA-MB-231) deployed by Zhang et al. primarily express collagen and laminin-binding $\beta_{1}$ integrin heterodi- mers $\left(\alpha_{2} \beta_{1}, \alpha_{1} \beta_{1}\right.$, and $\left.\alpha_{6} \beta_{1}\right)$, whereas the A2780 cells of the Caswell et al. study (7) mainly express $\alpha_{5} \beta_{1}$ : a key difference that may alter the way in which $\beta_{1}$ integrindependent RCP trafficking is targeted. Secondly, the type of RTK recruited to RCP will likely differ among cancer types, and this could influence whether ERK signaling is favored over PKB/AKT signaling or vice versa. Finally, the way in which RCP is mobilized to engage with trafficking RTKs is likely to influence signaling outcomes. RCP function and its ability to bind to $\alpha_{5} \beta_{1}$ and EGFR is enabled following occupation of another integrin $\left(\alpha_{v} \beta_{3}\right)$ with the prometastatic ligand osteopontin (7). However, expression of RAB25, which due to sequences present in its GTP-binding domain may be constitutively active, or expression of mutant forms of $\mathrm{p} 53$ can activate RCP trafficking in the absence of osteopontin, and it is likely that the car-

\section{Figure 1}

Role of RCP in integrin and RTK trafficking during tumor cell migration. (A) Activated RCP associates with $\beta_{1}$ integrin and acts to link this integrin with RTKs at recycling endosomes. The consequence of RCP-dependent corecycling of integrin and RTK is enhanced signaling, which drives cell proliferation and cell migration in $2 \mathrm{D}$ and $3 \mathrm{D}$ matrices. (B) RCP recruits a spatially restricted cycling population of receptors to the tips of invasive pseudopods during tumor cell migration in $3 \mathrm{D}$ microenvironments.

goes of RCP vary under these different contexts. It will be necessary to define not only the mechanisms inducing RCP "activation" but also the precise nature of the cargoes recruited to RCP in a range of cancer types and cancer-relevant situations. Further, the RAB11FIP-binding network integrates a complex matrix of RAB proteins, RAB-binding proteins, and vacuolar protein-sorting homologs as well as key motility and polarity factors such as PKC-ı and $-\zeta$ (Figure 2). Understanding how polarity, vesicle formation, exocytosis, endocytosis, and recycling of cargoes coordinate into appropriate cellular output in normal cells and when aberrant contribute to the hallmarks of cancer will require a major systems biology effort.

\section{Future directions}

Targeting RAS-superfamily members has proven frustrating. The association of RAB11 family members with membranes could potentially be targeted by geranylgeranyltransferase (GGTase) inhibitors. However, as a number of proteins are substrates for GGTases, it may be difficult to selectively target RAB11 and RAB11FIP family members. Although RAB GTPases have multiple regulators, none of these have proven amenable to targeting by small molecule inhibitors. The regulators target multiple RABs, and each RAB is targeted by multiple regulators (Figure 2), providing additional complexity. Although protein interactions have proven recalcitrant to targeting, fragment-based drug discovery and peptide stapling have the potential to provide effective approaches to targeting protein interactions (15). Thus, it may be possible to target the binding interface between RAB11 family members and RAB11FIPs (16). Recent studies suggest that siRNAs can be delivered to tumors in vivo in sufficient amounts to target molecules normally thought to be untargetable (17). 


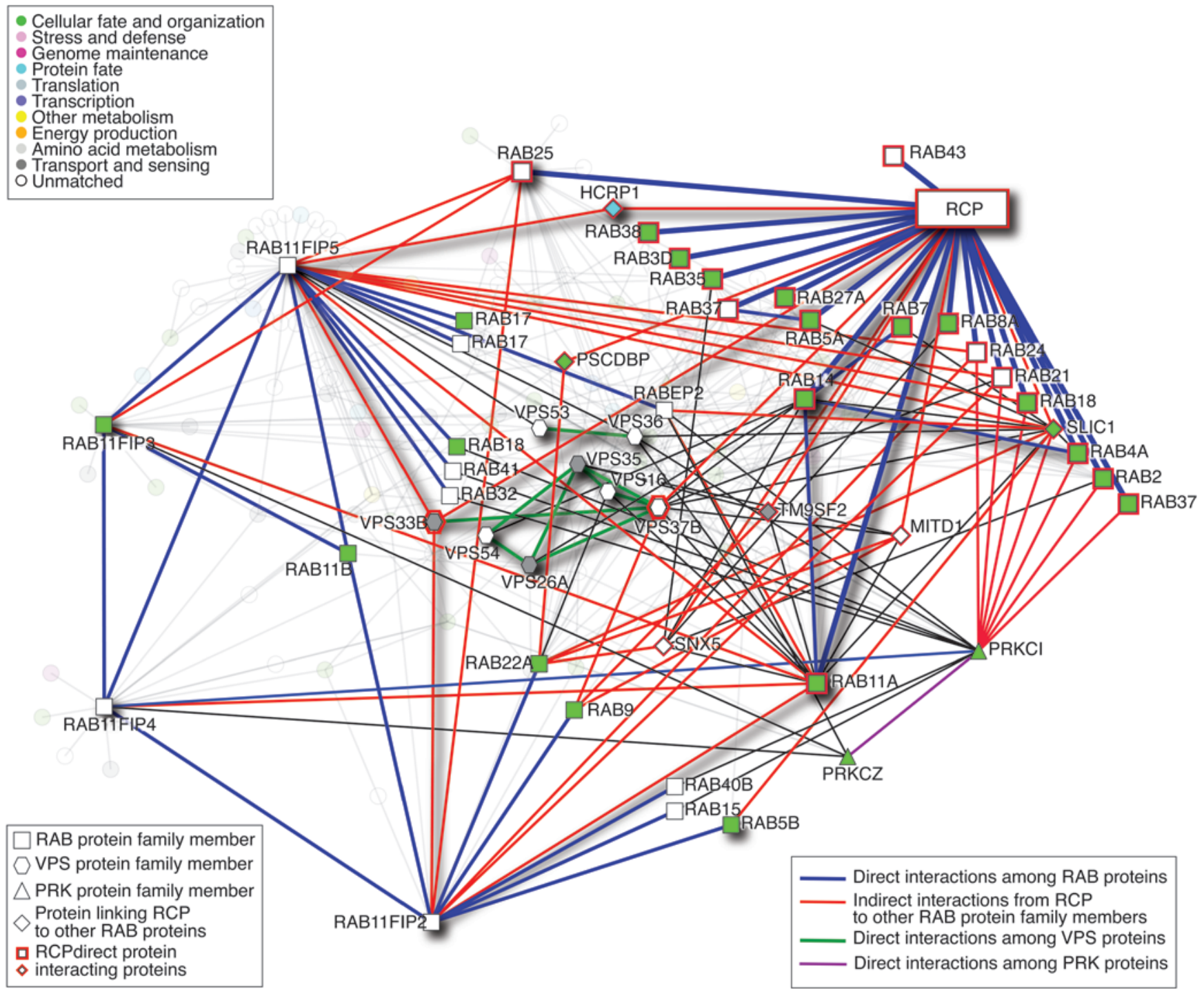

\section{Figure 2}

RCP protein-protein interaction network. RAB11FIP family members function by binding to multiple different RAB proteins to coordinate vesicle function and polarity. In order to effectively target the functions of RCP, it will be necessary to understand how the interaction of RCP with its partners regulates cellular functions. Using Interologous Interaction Database v1.71 (http://ophid.utoronto.ca/i2d), we constructed a protein-protein interaction network highlighting RCP interactions with other RAB and VPS (vacuolar protein sorting) family members. The network comprises 159 proteins and 447 physical protein interactions. Only direct, physical interactions were included, covering human-curated, high-throughput, and interologous interactions (individual interaction sources are listed at http://ophid.utoronto.ca/ophidv2.201/statistics.jsp). Protein function according to Gene Ontology (http://www.geneontology.org/), vesicle function, and polarity are highlighted according to the legends. Red-highlighted nodes represent direct physical interactions with RCP. RAB protein family members are shown as rectangles; blue edges correspond to direct interactions among RAB proteins. VPS protein family members are indicated by hexagons; green edges correspond to direct interactions among VPS proteins. PRK family proteins are triangle nodes; purple edges show direct interactions among PRK proteins. Black edges identify interactions that connect RAB, VPS, and PRK family proteins. Network visualization was done in NAViGaTOR, version 2.1.15 (http://ophid.utoronto.ca/navigator).

The current study by Zhang et al. (1) adds to a wealth of data implicating vesicle function, exocytosis, endocytosis, and vesicle recycling as new and critical hallmarks of cancer. Importantly, $R C P$ has oncogenelike characteristics and is likely a driver of the 8p11-12 amplicon. The observation that $R A B 25, R A B 23$, and $R C P$ are targeted by genomic amplicons, combined with the recent demonstration that GOLPH3, another vesicle regulator, is a driver of the $5 \mathrm{p} 13$ amplicon in multiple cancers (18) further supports the concept that vesicle function is a frequent target of amplicons in cancer.

\section{Acknowledgments}

G.B. Mills is supported by National Cancer Institute grant PO1CA099031. I. Jurisica is supported by Genome Canada. The authors wish to thank Greg Verdine, Roshan Agarwal, Kwai Wa Cheng, and Fan Zhang for figures, analysis of databases, and key discussions.

Address correspondence to: Gordon B. Mills, Department of Systems Biology, University of Texas, MDA.2SCR3.1030A, MD Anderson Cancer Center, 1515 Holcombe 
Blvd., Houston, Texas 77030, USA. Phone: (713) 563-4200; Fax: (713) 563-4235; E-mail: gmills@mdanderson.org.

1. Zhang, J., et al. 2009. RCP is a human breast can cer-promoting gene with Ras-activating function. J. Clin. Invest. 119:2171-2183.

2. Kauraniemi, P., Barlund, M., Monni, O., and Kallioniemi, A. 2001. New amplified and highly expressed genes discovered in the ERBB2 amplicon in breast cancer by cDNA microarrays. Cancer Res. 61:8235-8240.

3. Letessier, A., et al. 2006. Frequency, prognostic impact, and subtype association of $8 \mathrm{p} 12,8 \mathrm{q} 24$ $11 \mathrm{q} 13,12 \mathrm{p} 13,17 \mathrm{q} 12$, and $20 \mathrm{q} 13$ amplifications in breast cancers. BMC Cancer. 6:245.

4. Chin, K. 2006. Genomic and transcriptional aberrations linked to breast cancer pathophysiologies. Cancer Cell. 10:529-541.

5. Cheng, K.W., et al. 2004. The RAB25 small GTPase determines aggressiveness of ovarian and breast cancers. Nat. Med. 10:1251-1256.
6. Hou, Q., et al. 2008. Integrative genomics identifies $\mathrm{RAB} 23$ as an invasion mediator gene in diffuse-type gastric cancer. Cancer Res. 68:4623-4630.

7. Caswell, P.T., et al. 2008. Rab-coupling protein coordinates recycling of alpha5beta 1 integrin and EGFR1 to promote cell migration in 3D microenvironments. J. Cell Biol. 183:143-155.

8. Agarwal, R., Jurisica, I., Cheng, K.W., and Mills, G.B. 2009. The emerging role of the Rab25 small GTPase in cancer. Traffic. In press.

9. Chamberlain, M.D., et al. 2008. Disrupted Rab GAP function of the 885 subunit of phosphatidylinositol 3-kinase results in cell transformation. J. Biol. Chem. 283:15861-15868.

10. Cheng, J.M., Ding, M., Aribi, A., Shah, P., and Rao, K. 2006. Loss of RAB25 expression in breast cancer. Int. J. Cancer. 118:2957-2964.

11. Palmieri, D., Bouadis, A., Ronchetti, R., Merino, M.J., and Steeg, P.S. 2006. Rab11a differentially modulates epidermal growth factor-induced proliferation and motility in immortal breast cells. Breast Cancer Res. Treat. 100:127-137.

12. Mossesson, Y., Mills, G.B., and Yarden, Y. 2008.
Derailed endocytosis: An emerging feature of cancer. Nat. Rev. Cancer. 8:835-850.

13. Jones, M.C., Caswell, P.T., and Norman, J.C. 2006. Endocytic recycling pathways: emerging regulators of cell migration. Curr. Opin. Cell Biol. 18:549-557.

14. Caswell, P.T., et al. 2007. Rab25 associates with alpha5beta1 integrin to promote invasive migration in 3D microenvironments. Dev. Cell. 13:496-510.

15. Verdine, G.L., and Walensky, L.D. 2007. The challenge of drugging undruggable targets in cancer: lessons learned from targeting BCL-2 family members. Clin. Cancer Res. 13:7264-7270.

16. Eathiraj, S., Mishra, A., Prekeris, R., and Lambright, D.G. 2006. Structural basis for Rab11-mediated recruitment of FIP3 to recycling endosomes. J. Mol. Biol. 364:121-135.

17. Sanguino, A., Lopez-Berestein, G., and Sood, A.K. 2008. Strategies for in vivo siRNA delivery in cancer. Mini Rev. Med. Chem. 8:248-255.

18. Scott, K.L., et al. 2009. Golgi oncoprotein, GOLPH3, activates mTOR signaling in cancer and modulates sensitivity to rapamycin. Nature. 459:1085-1090.

\title{
Anticancer immunochemotherapy using adjuvants with direct cytotoxic effects
}

\author{
Laurence Zitvogel1,2,3,4 and Guido Kroemer ${ }^{3,4,5}$
}

\begin{abstract}
${ }^{1}$ INSERM U805, Villejuif, France. ${ }^{2}$ CIC BT507, Villejuif, France. 3Institut Gustave Roussy, Villejuif, France. ${ }^{4}$ Faculté Paris Sud-Université Paris 11, Kremlin-Bicêtre, France. ${ }^{5}$ INSERM U848, Villejuif, France.
\end{abstract}

\begin{abstract}
Conventional chemotherapeutics may induce immunogenic cancer cell death or stimulate immune effectors via so-called off-target effects. The study by Besch et al. in this issue of the JCI now demonstrates that agents designed to stimulate the innate immune system by activating intracellular pattern recognition receptors can kill cancer cells in a direct, cell-autonomous fashion (see the related article beginning on page 2399). The authors show that ligation of viral RNA sensors, such as RIG-I or MDA-5, by viral RNA mimetics triggers mitochondrial apoptosis in human melanoma cells in an IFN-independent fashion. The data suggest that tumor cell killing and immunostimulation may synergize for optimal anticancer immunochemotherapy.
\end{abstract}

Adjuvants are natural or synthetic compounds that stimulate the immune response, mostly by interacting with receptors of the innate immune system (i.e., pattern recognition receptors [PRRs]) that have evolved to recognize viral or bacterial

Conflict of interest: Laurence Zitvogel has received a research grant from Innate Pharma. Guido Kroemer has received a research grant and consultancy fees from Bayer Schering Pharma.

Nonstandard abbreviations used: IRF-3, IFN regulatory factor 3; MDA-5, melanoma differentiation-associated antigen 5; PAMP, pathogen-associated molecular pattern; PKR, RNA-activated protein kinase; poly(I:C), polyinosinic-polycytidylic acid; pppRNA, 5 '-triphosphate RNA; PRR, pattern recognition receptor; RIG-I, retinoic acid-inducible gene $I$.

Citation for this article: J. Clin. Invest. 119:2127-2130 (2009). doi:10.1172/JCI39991. structures known as pathogen-associated molecular patterns (PAMPs) (1). One of the hallmarks of cancer is the avoidance or suppression of antitumor immune responses (2), and the introduction of adjuvants into tumors, alone or in combination with cytotoxic agents, has been attempted in an effort to stimulate anticancer immune responses. Local instillation of bacterial extracts (e.g., Bacillus Calmette-Guérin) is now the standard therapy for noninvasive bladder cancer (3), and superficial basal cell carcinoma is usually eradicated by topical application of a synthetic PRR activator, imiquimod (which activates the PRR known as TLR7) (4). Innate effectors of the immune system (such as DCs) are activated by PAMPs to stimulate NK and
CTL responses. In addition, tumor cells that are exposed to PAMPs can produce chemokines and cytokines (such as type I IFNs), which attract immune effectors into the tumor bed (1). Most immunoadjuvants have been generated to stimulate surface-exposed or lysosomal TLRs, which constitute the first class of PRRs to be discovered. However, recent studies have been evaluating the possibility of targeting cytosolic PRRs that detect the presence of viral genomes in infected cells $(1,5)$. Endogenous RNA present in the cytoplasm is largely single stranded and lacks 5'-triphosphate RNA (pppRNA) moieties because the RNA transcribed from nuclear host DNA is processed to remove them (for instance by $5^{\prime}$ capping and splicing) before the RNA is exported from the nucleus. In contrast, viral RNA stimulates specific cytosolic receptors by virtue of the presence of $5^{\prime}$-triphosphate residues (which interact with the cytoplasmic PRR retinoic acid-inducible gene I [RIG-I]) or that of double-stranded structures (which are mostly recognized by a RIG-I homolog, melanoma differentiation-associated antigen 5 [MDA-5]) $(1,5)$. Upon recognition of viral RNA mimetics such as synthetic 